\title{
COMPUTABILITY OF FINITE QUOTIENTS OF FINITELY GENERATED GROUPS
}

\author{
EMMANUEL RAUZY
}

\begin{abstract}
We study systematically groups whose marked finite quotients form a recursive set. We give several definitions, and prove basic properties of this class of groups, and in particular emphasize the link between the growth of the depth function and solvability of the word problem. We give examples of infinitely presented groups whose finite quotients can be effectively enumerated. Finally, our main result is that a residually finite group can be even not recursively presented and still have computable finite quotients, and that, on the other hand, it can have solvable word problem while still not having computable finite quotients.
\end{abstract}

\section{INTRODUCTION}

The fact that several well known conjectures which have been solved for countable groups remain open for finitely presented groups, such as the Burnside problem, or the existence of groups of intermediate growth, shows that little is known about the specificities of finitely presented groups.

One of the most striking results that affects specifically finitely presented groups is McKinsey's theorem : finitely presented residually finite groups must have solvable word problem. (McKinsey's theorem, which appeared in [19], is in fact set in a more general setting than that of finitely presented residually finite groups, we are only interested in its group theoretical version, which was first made explicit by Mal'cev in [18, followed by Dyson in [7] and by Mostoswski in [21.) The proof of this theorem hinges on the fact that the finite quotients of a finitely presented group can be enumerated. However, it is known that recursively presented residually finite groups can have unsolvable word problem, and thus quotients that cannot be enumerated: two examples of these exist in the literature, one by Meskin $([20])$, which is in addition center-by-metabelian, and one by Dyson in [8]. This proves in particular that there can be no Higman theorem for general residually finite groups: not all recursively presented residually finite groups embed in finitely presented residually finite groups.

Before stating a precise definition of "having computable finite quotients", let us recall the proof of McKinsey's theorem.

Consider a finitely presented residually finite group $G$, with a generating family $S$ of cardinal $n$, and $w$, a word whose letters are elements of $S \cup S^{-1}$. We try to determine whether $w=e$ in $G$.

First, as in any recursively presented group, we can apply to $w$ an algorithm that will stop if it is the identity element of the group, and that never stops otherwise. This is done by enumerating relations, and their conjugates, and the products of their conjugates, and checking every time whether the word $w$ has appeared. 
Secondly, notice that if $F$ is a finite group, one can determine in a finite number of steps whether $F$ is a quotient of $G$ : this is done by checking, for every generating family of $F$ of cardinal $n$, whether the (finitely many) defining relations of $G$ hold between those generators. Thus, from an enumeration of all finite groups by their Cayley table, one can obtain an enumeration of all finite quotients of $G$. In each of those quotients, we can check whether or not the image of $w$ is trivial. If a quotient is found, in which the image of $w$ is different from the identity, we can conclude that in $G$ as well $w$ must be different from the identity, and stop that procedure. By definition of a residually finite group, any non-trivial element of $G$ will have a non-trivial image in a finite quotient, thus that second part of the algorithm will always stop if $w$ is not the identity element in $G$.

This proof is the sum of three facts:

(1) In a recursively presented group, there is an algorithm that determines when a word corresponds to the identity (and never stops otherwise).

(2) In a finitely presented group, there is an algorithm that determines when a finite group is a quotient of it, and produces a morphism.

(3) In a residually finite group whose finite quotients can be enumerated, there is an algorithm that determines when a word corresponds to a non-identity element (and never stops otherwise).

The first point is an equivalence and is well known. The last point is very natural, and the definition of "residually finite group" could have been introduced to answer the question: "what is a sufficient condition for a group whose finite quotients can be enumerated to have solvable word problem?".

The second point is the one at the origin of this article, which consists in a systematic study of groups whose finite quotients can be detected. Although several articles $([4,9,13])$ have already mentioned the fact that it is sometimes possible to enumerate the finite quotients of non-finitely presented groups, the following was never stated as a definition:

Definition 1. A finitely generated group $G$, together with a generating set $S$, is said to have Computable Finite Quotients (CFQ) if there is an algorithm that, given a pair $(F, f)$, where $F$ is a finite group (given by a finite presentation) and $f$ is a function from $S$ to $F$, determines whether the function $f$ extends to a group morphism, that is whether there exists a group homomorphism $\hat{f}: G \rightarrow F$ such that for any $s$ in $S, f(s)=\hat{f}(s)$.

If there exists an algorithm that terminates when the function $f$ extends to a group morphism, but does not terminate otherwise, we say that $G$ has Recursivelyenumerable Finite Quotients (ReFQ).

If there exists an algorithm that terminates when the function $f$ does not extend to a group morphism, but does not terminate otherwise, we say that $G$ has coRecursively-enumerable Finite Quotients (co-ReFQ).

Of course having CFQ is equivalent to having both ReFQ and co-ReFQ.

It follows from the proof given earlier of McKinsey's algorithm that all finitely presented groups have CFQ. This fact should be compared to a result of Bridson and Wilton from [5]: there is no algorithm that, given a finite presentation, decides whether or not the group it defines admits a non-trivial finite quotient.

This article builds upon Dyson's groups from [8] to obtain the following two theorems: 
Theorem 2. There exists a finitely generated residually finite group with solvable word problem, but that has uncomputable finite quotients.

Theorem 3. There exists a finitely generated residually finite group with computable finite quotients, that still has unsolvable word problem.

We proceed as follows.

In the first section, we give several equivalent definitions of CFQ groups, and we explain how the profinite topology on a group can be used to describe the various decisions problems that can be solved using McKinsey-type algorithms. The second section quickly enumerates some easy properties: free or direct products of groups with computable finite quotients also have this property, etc. In the third section interactions with the depth function for residually finite groups are explained. The fourth section provides examples of infinitely presented groups with CFQ: wreath products of groups with CFQ and L-presented groups (including some well known torsion groups). In the final section, we prove Theorem 2, thanks to Dyson's method which uses doubles of the lamplighter group, and give two proofs of Theorem 3 , one using the same technique, and another one that relies on Slobodskoi's work in [23.

Following [8], throughout this article, recursively presented groups will be called re groups (for recursively enumerable), and groups in which there is an algorithm that recognizes non-trivial elements will be called co-re groups. A group has solvable word problem if and only if it is re and co-re.

\section{CFQ Groups}

1.1. Equivalent definitions. Just as a group with solvable word problem is a group in which words in the generators corresponding to the identity can be enumerated by an algorithm which respects a computable ordering on the set of words in the generators, or a re group is a group in which these words can be enumerated, but without any guarantee on the order of the enumeration, groups with CFQ, ReFQ or co-ReFQ can be equivalently characterized by enumeration of their finite quotients. Let us precise this.

$G$ is still a group generated by $S$, of cardinal $n$. $S$ can be seen as $\{1, \ldots, n\}$.

Call a $n$-marked finite group a pair $(F, f)$, where $F$ is a finite group given by its Cayley table, and $f$ is a function from $\{1, \ldots, n\}$ to $F$ whose image generates all of $F$. Consider an effective enumeration $\left(F_{1}, f_{1}\right),\left(F_{2}, f_{2}\right),\left(F_{3}, f_{3}\right), \ldots$ of all $n$-marked finite groups, which satisfies $\operatorname{card}\left(F_{n}\right) \leq \operatorname{card}\left(F_{n+1}\right)$. (This can be obtained by listing in order all possible Cayley tables, then listing all $n$-tuples from those tables and determining when a tuple defines a generating set). Define $\mathcal{A}_{G} \subseteq \mathbb{N}$ to be the set of indices $k$ for which $f_{k}$ defines a morphism from $G$ to $F_{k}$. Then $G$ has CFQ, ReFQ or co-ReFQ if and only if $\mathcal{A}_{G}$ is, respectively, a recursive set, a recursively enumerable set or a co-recursively enumerable set.

Of course, those definitions are independent of a choice of a generating family.

Proposition 4. Having one of $C F Q, R e F Q$ or co-ReFQ is independent of a choice of a generating family.

Proof. Let $S$ and $T$ be two finite generating sets of a group $G$ (not necessarily of the same cardinal). Fix for each $s$ in $S$ an expression $s=t_{1}^{\alpha_{1}} \ldots t_{k}^{\alpha_{k}}$, with $\alpha_{i} \in\{-1,1\}$ and $t_{i} \in T$, that gives $s$ as a product of elements of $T$ or their inverses, and for each $t$ in $T$ an expression $t=s_{1}^{\beta_{1}} s_{2}^{\beta_{2}} \ldots s_{k}^{\beta_{k}}$ that describes $t$ in terms of the generators of 
$S$ and their inverses. For a finite group $F$ and a function $f$ from $S$ to $F$, define the function $f^{\prime}$ from $T$ to $F$ by $f^{\prime}(t)=f\left(s_{1}\right)^{\beta_{1}} \ldots f\left(s_{k}\right)^{\beta_{k}}$. The function $f$ defines a homomorphism if, and only if, $f^{\prime}$ also defines a homomorphism $\varphi^{\prime}$, that satisfies $\varphi^{\prime}(s)=f(s)$ for $s$ in $S$. That last condition is an equality in $F$ that can be tested using the expressions $s=t_{1}^{\alpha_{1}} \ldots t_{k}^{\alpha_{k}}$, even before it is known whether or not $f^{\prime}$ extends. Using this, all three properties, CFQ, ReFQ, co-ReFQ can be seen to be independent of the chosen generating family of $G$.

1.2. Variations on McKinsey's algorithm and the profinite topology. It was already explained, when discussing McKinsey's original algorithm, that the notion of residually finite group becomes a very natural one to introduce when one asks for a sufficient condition for a group with CFQ to be co-re. We can more generally search for conditions on a finitely generated group $G$ with CFQ, that might allow to solve various algorithmic problems. We will see that such conditions can be expressed through the use of the profinite topology on $G$. In what follows, we will say that we use McKinsey's algorithm to mean that we enumerate all finite quotients of a group, checking some condition in each quotient, and stopping when a finite quotient is found that satisfies the required condition.

Fix a finitely generated group $G$ with CFQ (or simply ReFQ).

We will search for conditions that allow, given two disjoint subsets $A$ and $B$ of $G$, to distinguish $A$ from $B$ using McKinsey's algorithm, that is to say to decide, given an element of $G$ that belongs to $A \cup B$, whether it belongs to $A$ or to $B$.

The first condition we need is that, given a morphism from $G$ onto a finite group $F$, it be possible to completely determine the images of $A$ and of $B$ in $F$. We will thus need the following definition:

Definition 5. A subset $A$ of $G$ is said to be determinable in finite quotients of $G$ if there exists an algorithm that, given a morphism $\phi$ from $G$ onto a finite group $F$, can determine the image $\phi(A)$ (i.e. solve the membership problem for $\phi(A)$ in $F)$.

A family $\left(A_{i}\right)_{i \in \mathbb{N}}$ of subsets of $G$ is uniformly determinable in finite quotients of $G$ if each $A_{i}$ is determinable in finite quotients of $G$, and if the algorithm that determines $A_{i}$ in finite quotients of $G$ depends recursively of $i$.

Note, as an example, that a finitely generated subgroup $H$ of a group $G$ is determinable in the finite quotients of $G$, because the image of $H$ in a quotient of $G$ is the group generated by the images of the generators of $H$.

The property of being determinable in finite quotients is interesting in itself, however we will not give it much attention in this paper. We still remark the following.

It is easy to build a family $\left(A_{i}\right)_{i \in \mathbb{N}}$ of subsets of $\mathbb{Z}$, such that: it is uniformly recursively enumerable, but not uniformly determinable in finite quotients of $\mathbb{Z}$. But a stronger result will naturally appear in this paper as a byproduct of the proof of Theorem 2 .

Proposition 6. There exists a recursive subset of $\mathbb{Z}$ that is not determinable in finite quotients of $\mathbb{Z}$.

Proof. See Lemma 33 disregarding the statement about the subset of $\mathbb{Z}$ being closed. 
On the other hand, it is easy to build a subset of $\mathbb{Z}$ that is determinable in its finite quotients, but not recursive.

Proposition 7. There exists a subset of $\mathbb{Z}$ that is not re, but still is determinable in finite quotients of $\mathbb{Z}$.

Proof. For a function $h$ that grows faster than any recursive function, consider the enumeration $2 h(1), 2 h(2)+1,3 h(3), 3 h(4)+1,3 h(5)+2,4 h(6), \ldots$ This defines a set that is not re, but whose image in any quotient $\mathbb{Z} / n \mathbb{Z}$ of $\mathbb{Z}$ is all of $\mathbb{Z} / n \mathbb{Z}$.

Given two disjoint subsets $A$ and $B$ of $G$ that are indeed determinable in finite quotients of $G$, the profinite topology of $G$ can be used to decide whether McKinsey's algorithm can tell them apart.

The profinite topology on a group $G$ was introduced in [11, it is the topology whose open basis consists of cosets of finite index normal subgroups of $G$. We denote $\mathcal{P} \mathcal{T}(G)$ the profinite topology on $G$. A closed subset of $G$ in $\mathcal{P} \mathcal{T}(G)$ is called a separable set. We note $\bar{A}$ the closure of a set $A$ in $\mathcal{P} \mathcal{T}(G)$. The following easy facts render explicit the link between the profinite topology and McKinsey-type algorithms:

Fact 8. A subset $A$ of a group $G$ is open in $\mathcal{P} \mathcal{T}(G)$ if and only if for any element a of $A$, there is a morphism $\phi$ from $G$ onto a finite group $F$ such that $\phi^{-1}(\phi(a)) \subseteq A$, i.e. such that if an element of $G$ has the same image as a in $F$, it also belongs to $A$.

Fact 9. The closure $\bar{B}$ of a subset $B$ of $G$ is the biggest set of elements that satisfy the following condition:

For any morphism $\phi$ from $G$ onto a finite group $F, \phi(\bar{B}) \subseteq \phi(B)$.

This shows that the closure of a set $B$ in $\mathcal{P T}(G)$ is precisely the set of elements that cannot be distinguished from $B$ using McKinsey's algorithm.

We can now give conditions that allow two disjoint subsets $A$ and $B$ of $G$ to be distinguishable by McKinsey's algorithm.

Proposition 10. Let $A$ and $B$ be disjoint subsets of $G$ that are determinable in finite quotients of $G$. Then McKinsey's algorithm can be used to distinguish A from $B$ if and only if the following two conditions hold:

$$
\begin{aligned}
& A \cap \bar{B}=\varnothing \\
& \bar{A} \cap B=\varnothing
\end{aligned}
$$

Proof. This is a simple consequence of Fact 9

McKinsey's original result on residually finite groups can thus be interpreted as an application of this proposition to singletons, and a residually finite group is precisely a group in which all the singletons are closed in the profinite topology.

Two other well studied families of groups fall in the range of Proposition 10 conjugacy separable groups, and LERF groups.

A conjugacy separable group is a group $G$ in which all the conjugacy classes are separable. It is easy to see that the conjugacy classes of a finitely generated group $G$ are always uniformly determinable in finite quotients of $G$ (when a class 
$C$ is given by any of its elements), thus Proposition 10 can be used to distinguish conjugacy classes.

A LERF group (for locally extended residually finite), or subgroup separable group, is a group $G$ whose finitely generated subgroups are separable.

We then have the following proposition:

Proposition 11. Let $G$ be a finitely generated group with ReFQ.

- If $G$ is residually finite, then it is co-re. If it is re and residually finite, it has solvable word problem.

- If $G$ is conjugacy separable, then there exits an algorithm that decides when two of its elements are not conjugate. If it is re and conjugacy separable, then it has solvable conjugacy problem.

- If $G$ is LERF, there is an algorithm that, given a tuple $\left(x_{1}, \ldots, x_{n}, g\right)$ of elements of $G$, stops exactly when $g$ does not belong to the subgroup of $G$ generated by $\left(x_{1}, \ldots, x_{n}\right)$. If it is re and LERF, it has solvable generalized word problem.

Proof. All three points follow from Proposition 10, noticing that it provides a uniform algorithm, and using it respectively with:

- $A$ and $B$ being singletons,

- $A$ and $B$ being conjugacy classes,

- $A$ being a finitely generated subgroup of $G$ and $B$ a singleton.

In each case, one needs to use the fact that those sets are uniformly determinable in finite quotients, which is straightforward.

Because re groups naturally have co-ReFQ (Proposition 16), the statements that concern re group in the previous proposition could be formulated with CFQ instead of ReFQ, without loss of generality.

Proposition 11 follows in a very straightforward way from the definitions of residually finite, of conjugacy separable and of LERF groups, and it is surprising that the study of these properties was not followed by a systematic study of the properties CFQ and ReFQ. The author could point the lector to papers where it is implied that re conjugacy separable groups always have solvable conjugacy problem, which led him to believe it has to be ascertained that not all recursively presented groups, or residually finite groups, or even residually finite groups with solvable word problem, have ReFQ.

1.3. Membership problem for finite index normal subgroups. In the article 4], Bou-Rabee and Seward use, in the course of a proof (4], Proof of Theorem 2), the fact that, if a group $G$ has solvable "membership problem for finite index normal subgroups", (or "generalized word problem for finite index normal subgroups") and solvable word problem, then it admits an algorithm that recognizes its finite quotients.

We will now show that for re groups, having solvable membership problem for finite index normal subgroups is actually equivalent to having CFQ. This will allow us to give another point of view on groups with CFQ, and at the same time making explicit the link to the isomorphism problem for finite groups given by recursive presentations, which we will sum up in the next sub-section.

When formulating the membership problem for finite index normal subgroups, it is implicit that the normal subgroup is given by a finite generating family. 
Indeed, the membership problem for finite index normal subgroups in a group $G$ asks for an algorithm that, given a tuple $\left(x_{1}, \ldots, x_{k}, g\right)$ of elements of $G$, the first $k$ elements of which generate a finite index normal subgroup of $G$, will decide whether $g$ belongs to that subgroup. As opposed to that, when working with property CFQ, we describe non-ambiguously a finite index normal subgroup $N$ of $G$ by a pair $(F, f)$, where $F$ is a finite group and $f$ a function from the generators of $G$ to $F$, which extends to a group homomorphism, the kernel of which is precisely $N$.

Of course, given that second description, the problem "does $g$ belong to $N$ " is solved by computing the image of $g$ in $F$ to see whether it is the identity of $F$. Thus a group in which one can go from the description of a normal subgroup by generators to a description of this subgroup by a morphism necessarily has solvable membership problem for finite index normal subgroups. We will see that for re groups this is also sufficient.

On the other hand, given a description by morphism of the subgroup $N$, that is a morphism $\varphi: G \rightarrow F$ with $\operatorname{ker} \varphi=N$, one can always obtain a description of it by generators, as one can effectively carry out the well known proof of Schreier's lemma, which is often used to prove that a finite index subgroup of a finitely generated group is itself finitely generated. Indeed, if $S$ is a generating family of $G$, for any $x$ in $F$ and $s$ in $S$, a preimage $\hat{x}$ of $x$ can be found in $G$, by exhaustive search, and a preimage of $x \varphi(s)$ can be found as well, call it $\hat{y}$. Schreier's lemma asserts that the elements of the form $\hat{x} s \hat{y}^{-1}$ generate $N$.

This allows us to prove the following (the backward implication is directly adapted from [4]):

Proposition 12. Property ReFQ is equivalent to having co-re membership problem for finite index normal subgroups, that is to having an algorithm that decides when an element is not in a given finite index normal subgroup, and does not terminate otherwise.

Proof. Suppose first that $G$ has ReFQ, and let $N$ be a finite index normal subgroup of $G$ generated by a family $x_{1}, \ldots, x_{k}$. Let finally $g$ be an element of $G$, we want to decide whether $g$ belongs to $N$. Enumerate the quotients $(F, f)$ of $G$, and look for a finite quotient in which the image of $g$ is non-trivial, while the images of $x_{1}, \ldots, x_{n}$ are all trivial. If $g$ does not belong to $N$, such a quotient exists (the projection $G \rightarrow G / N)$, and this algorithm will terminate.

Now suppose $G$ has co-re membership problem for finite index normal subgroups. Write $\langle S \mid R\rangle$ a presentation of $G$. Let $(F, f)$ be a finite group together with a function from $S$ to $F$. As $f$ does not necessarily define a morphism, we cannot yet apply Schreier's method. But if $\mathcal{F}_{n}$ is a free group with basis the $n$ generators of $G, f$ does define a morphism $\varphi$ from $\mathcal{F}_{n}$ to $F$, and thus we can find a family $x_{1}, \ldots, x_{k}$ of elements of $\mathcal{F}_{n}$ that generate $\operatorname{ker}(\varphi) . F$ is given by the presentation: $\left\langle S \mid x_{1}, \ldots, x_{k}\right\rangle$. (But $x_{1}, \ldots, x_{k}$ generate $\operatorname{ker} \varphi$ as a group, and not only as a normal subgroup as would be guaranteed by any presentation of $F$ on the generators $S$ ).

Now $f$ extends to a morphism if and only if $F$ satisfies all the relations of $G$, that is to say if and only if the relations $x_{1}, \ldots, x_{k}$ imply the relations that appear in $R$, that is to say if and only if $\left\langle S \mid R, x_{1}, \ldots, x_{k}\right\rangle$ is just another presentation of $F$. But this is a presentation of $G / N$, where $N$ is the subgroup of $G$ generated by $x_{1}, \ldots, x_{k}$. If $f$ does not extend to a morphism, $G / N$ is a strict quotient of $F$.

Thus we can do the following: enumerate the elements of $G, g_{1}, g_{2}, \ldots$ Then use the membership algorithm for $N$, (which, as we suppose, can only show something 
does not belong to $N$ ), to find elements that define different classes in $G / N$, that is: find $g_{i_{0}}$ that does not belong to $N$, then $g_{i_{1}}$ which is such that neither itself nor $g_{i_{0}} g_{i_{1}}^{-1}$ belong to $N$, and $g_{i_{2}}$ such that $g_{i_{2}}, g_{i_{0}} g_{i_{2}}^{-1}$ and $g_{i_{1}} g_{i_{2}}^{-1}$ don't belong to $N \ldots$ If $F$ is a quotient of $G$, this method will yield $\operatorname{card}(F)$ elements, at which point the algorithm has proven that $F$ is a quotient of $G$. Of course, if $F$ is not a quotient of $G$, it will never stop.

In a re group, determining whether $g$ belongs to the subgroup generated by $x_{1}, \ldots, x_{k}$ can always be done when $g$ belongs to that group, thus having co-re membership problem for finite index normal subgroups is equivalent to having solvable membership problem for finite index normal subgroups. Similarly, re groups always have co-ReFQ (Proposition 16), thus for such a group ReFQ and CFQ are equivalent. This yields:

Corollary 13. For recursively presented groups, having CFQ and having solvable membership problem for finite index normal subgroups are equivalent properties.

We can use this to show that in a re group with $\mathrm{CFQ}$, from the description of a finite index normal subgroup $N$ by a generating family $x_{1}, \ldots, x_{k}$, one can deduce a pair $(F, f)$, where $F$ is a finite group and $f$ extends to a morphism $\varphi$ of $G$ to $F$ with kernel $N$.

Launch two procedures, one is the same as that described in the proof above: enumerate elements of $G$ that define different cosets of $G / N$. We get successively better lower bounds on $\operatorname{card}(G / N): \operatorname{card}(G / N) \geq 1,2,3, \ldots$

The other procedure gives upper bounds on the size of $G / N$. Start from the enumeration of all marked finite groups $\left(F_{1}, f_{1}\right),\left(F_{2}, f_{2}\right), \ldots$ For each pair $\left(F_{i}, f_{i}\right)$, test whether $G / N$ is a quotient of the group $F_{i}$ according to one of the finitely many left inverses of $f_{i}$. This can be done because $G / N$ is given by a recursive presentation (as we add finitely many relations to a presentation of $G$ which we suppose re), thus there is an algorithm that tests whether the finitely many relations of a finite group $F$ are satisfied in $G / N$, and terminates when indeed they are. This procedure yields upper bounds on the cardinal of $G / N$.

At some point, the lower and upper bounds will agree, and we will know that the pair $(F, f)$ that has $\operatorname{card}(F)=\operatorname{card}(G / N)$ defines an isomorphism $F \simeq G / N$, and thus the normal subgroup $N$ is described by the pair $(F, f)$.

1.4. Isomorphism problem for finite groups. Note that another condition for CFQ appears clearly in the course of the proof of Proposition 12 at some point, it is known that the presentation $\left\langle S \mid R, x_{1}=e, \ldots, x_{k}=e\right\rangle$ is the presentation of a finite group (even, that it is a quotient of the given group $F$ ), and the question "is $F$ a quotient of $G$ " is equivalent to "is this finite group a strict quotient of $F$ ". It follows from this remark:

Proposition 14. A group $G$, which admits a presentation $\langle S \mid R\rangle$, has $C F Q$ if the isomorphism problem is solvable for the following family of presentations: all finite presentations of finite groups, and all presentations of the form $\left\langle S \mid R, R_{1}\right\rangle$, where $R_{1}$ is a finite set of relations such that $\left\langle S \mid R_{1}\right\rangle$ is finite.

The isomorphism problem for finite groups is solvable, this is well known, but it only means that we can determine when two finite groups given by finite presentations are isomorphic, and the question here is to determine whether these two 
groups, one given by a finite presentation, and the other one by an infinite presentation, are isomorphic. It can be seen that the finite presentations of finite groups can actually be omitted in Proposition 14. If $G$ is a re group which does not have CFQ (and such groups exist, see Theorem 21), this shows in particular:

Corollary 15. There exists a recursive family of recursive presentations of finite groups, for which the isomorphism problem is unsolvable. Moreover any two of those presentations differ only by a finite number of relations.

Since it was remarked that it suffices to be able to obtain lower bounds of the cardinal of the groups given by these presentations to solve their isomorphism problem, the world problem is not uniformly solvable for this family of presentation.

\section{BASIC PROPERTIES}

We will now quickly establish some basic results about the three properties CFQ, ReFQ and co-ReFQ.

2.1. Recursively presented groups. It is easy to see that re groups have coReFQ.

Proposition 16. Any recursively presented group has co-ReFQ.

Proof. Let $G$ be a re group generated by $S$ of cardinal $n$. Let $(F, f)$ be a $n$-marked finite group. For any relation $r$ of $G$, write $r=s_{1}^{\alpha_{1}} \ldots s_{k}^{\alpha_{k}}$ with $\alpha_{i} \in\{-1 ; 1\}$ and $s_{i} \in S$, we again test the equality $e=f\left(s_{1}\right)^{\alpha_{1}} \ldots f\left(s_{k}\right)^{\alpha_{k}}$. Since we suppose $G$ re, this can be carried out successively on all relations of $G$. If $f$ does not extend to a morphism, a relation true in $G$ but not in $F$ will eventually be found.

This is remarkable because it is more often the case that algorithmic problems for groups be naturally re for re groups, than co-re (conjugacy problem, generalized word problem, isomorphism problem for finitely presented groups, etc).

\subsection{Hereditarity.}

Proposition 17. If $G$ and $H$ both have one of $C F Q$, ReFQ, co-ReFQ, then so does their free product $G * H$.

Proof. Note that we have shown that those properties are independent of the generating family, thus we can show this using as a generating family of $G * H$ the union of a generating family of $G$ and of one of $H$. The proof is then straightforward, as a function from that generating family to a finite group extends to a morphism of $G * H$, if and only if both restrictions to $G$ and to $H$ extend as morphisms.

Proposition 18. ReFQ is inherited by finite index subgroups.

Proof. Let $G$ be a group, and $N$ a finite index subgroup of $G$. An enumeration of the finite quotients of $G$ gives an enumeration of finite quotients of $N$, by restricting the homomorphisms to $N$. Not all quotients of $N$ need arise this way, but add the following: whenever a pair $(F, f)$ is found that defines a quotient of $N$, list all quotients of the finite group $F$, and when a quotient $F \stackrel{\pi}{\rightarrow} F_{0}$ is found, add $\left(F_{0}, \pi \circ f\right)$ to the list of quotients of $N$. We claim that all finite quotients of $N$ arise this way.

Let $M$ be a finite index normal subgroup of $N$. Then $M$ is of finite index in $G$, it may not be normal in $G$, but it contains a normal subgroup $M_{0}$ which is both finite 
index and normal in $G$. Then $G \rightarrow G / M_{0}$ restricts to a morphism $N \rightarrow N / M_{0}$, and $M / M_{0}$ is a normal subgroup in $N / M_{0}$, and the quotient of $N / M_{0}$ by $M / M_{0}$ is, of course, $N / M$.

Note that any countable group embeds in a two generated simple group, and that simple groups always have CFQ. This of course shows that CFQ is not inherited by subgroups. Note that the author doesn't know of a residually finite group with CFQ, with a subgroup without $\mathrm{CFQ}$.

Problem 19. Find a finitely presented residually finite group with a finitely generated subgroup that does not have CFQ.

This problem is relevant in the search of a corrected Higman Embedding Theorem for residually finite groups, i.e. in the search of necessary and sufficient conditions for a finitely generated residually finite group to be a subgroup of a finitely presented residually finite group.

The following proposition provides insight into what happens when computing the quotient of a group with CFQ.

Proposition 20. Let $G$ be a finitely generated group. Let $H$ be a group obtained by adding finitely many relations and identities to $G$. If $G$ has any of $\operatorname{Re} F Q, c o-R e F Q$ or $C F Q$, then so does $H$.

Proof. Let $G$ and $H$ be two finitely generated groups, with a morphism $\pi$ from $G$ onto $H$. Let $(F, f)$ be a marked finite group. It is obvious that if $f$ extends to a homomorphism $\varphi$ from $H$ onto $F$, then $f \circ \pi$ extends as well to a morphism $\psi$, which, in addition, satisfies $k \operatorname{er}(\pi) \subseteq \operatorname{ker}(\psi)$. On the other hand, if $f \circ \pi$ extends to a morphism $\psi$, and if $\operatorname{ker}(\pi)$ is contained in $\operatorname{ker}(\psi)$, then $\psi$ factors through $\pi$ and $f$ will extend to a morphism. The diagram is the following:

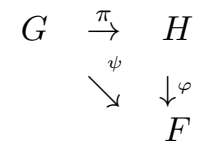

Thus if $G$ has CFQ or ReFQ, we can reduce the finite quotient question of $H$ to a question about subgroups inclusion.

If $\operatorname{ker}(\pi)$ is finitely generated as a normal subgroup, then the question "is $\operatorname{ker}(\pi)$ contained in $\operatorname{ker}(\psi)$ ?" can be solved in finite time, as it is solved by computing $\psi(r)$ for each $r$ in a generating family of $\operatorname{ker}(\pi)$. If $\operatorname{ker}(\pi)$ is generated by an identity -that is a set of relations of the form $v\left(x_{1}, \ldots, x_{k}\right)$, where $v$ is a element of the free group on $k$ generators, and $x_{1}, \ldots, x_{k}$ take all possible values of $G^{k}$ - this question can also be answered, because to check whether an identity holds in a finite group, one only needs to check finitely many relations.

If $G$ is a co-ReFQ group, we can determine whether $\operatorname{ker}(\pi)$ is contained in $\operatorname{ker}(\psi)$ even without knowing if $\psi$ defines a morphism from $G$, and thus if $(F, f)$ does not define a quotient of $H$, we will either prove that $f \circ \pi$ does not extend to a quotient of $G$, or that, even if it were to define a quotient, the inclusion of kernels would not hold. With this, all cases of Proposition 20 are covered.

Since free groups obviously have CFQ, this proves again that finitely presented groups have CFQ, and the improvement due to Mostowski [21] which asserts that groups defined by finitely many relations and identities have CFQ. This implies for 
instance that all finitely generated metabelian groups, while not being necessarily finitely presented, have CFQ, because a result of Hall ([12]) implies that any finitely generated metabelian group can be presented with the metabelian identity $(\forall x \forall y \forall z \forall t[[x, y][z, t]]=e)$ together with finitely many relations.

The following is a direct consequence of Proposition 20.

Corollary 21. The fundamental group of a graph of groups with vertex groups that have $C F Q$ (or ReFQ or co-ReFQ) and finitely generated edge groups also has $C F Q$ (respectively $R e F Q$ or co-ReFQ). This includes free products amalgamated over finitely generated subgroups and HNN extensions over finitely generated subgroups.

$A$ direct product of groups that have one of $C F Q, R e F Q$ or co-ReFQ, again has that property.

2.3. Groups with the same finite quotients. Although having CFQ is defined for any finitely generated group, this property is very much attached to residually finite groups, and not only because of the interaction between having CFQ and having solvable word problem. For a group $G$, define its finitary image (the name comes from [8]) to be the quotient of $G$ by its finite residual, i.e. by the intersection of all its finite index subgroups, or again, the image of $G$ in its profinite completion $\hat{G}$. Note this group $G_{f}$. It is the biggest residually finite quotient of $G$. Note $\pi$ the morphism $G \rightarrow G_{f}$. Fix a finite marked group $(F, f)$. This is the same situation as described when investigating quotients of CFQ groups, except that we have the property, which follows from the universal property of $G_{f}$ : any morphism $\psi$ of $G$ to a finite group $F$ factors through $\pi$, that is for any morphism $\psi$ to a finite group, $\operatorname{ker}(\pi) \subseteq \operatorname{ker}(\psi)$. The situation is summed up in the diagram:

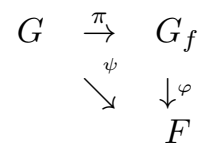

It follows that $G$ has any of CFQ, ReFQ, co-ReFQ if and only if $G_{f}$ shares the same property. From this, the following proposition is obvious.

Proposition 22. If two finitely generated groups $G$ and $H$ admit the same finitary image, then $G$ has any of $C F Q, R e F Q$, co-ReFQ if and only if $H$ shares the same property.

We say that a group has trivially computable finite quotients if its finitary image is a finite group. For instance, simple groups, or finite exponent groups (because of the solution to the restricted Burnside problem), all have trivially CFQ. Note again that since a finitely generated simple group can be neither re nor co-re, CFQ groups can have as bad algorithmic properties as desired. Obtaining groups with CFQ that still have bad algorithmic properties becomes challenging only amongst residually finite groups. This is what was obtained in Theorem 3 .

In [8] are constructed two groups with solvable word problem, the finitary image of one is co-re but not re, while the finitary image of the second is re but not co-re. This last group cannot have CFQ, otherwise its finitary image would have CFQ while being residually finite, thus it would be co-re. Thus this is a first example of a group with solvable word problem but without CFQ. Note however that this construction relies on the fact that the constructed group is not residually finite in 
order to prove that it does not have CFQ; while Theorem 2 provides a group with solvable word problem and without CFQ that is itself residually finite.

\section{Relation with the Depth Function for Residually finite groups}

In [3], Bou-Rabee introduced the residual finiteness growth function, or depth function, $\rho_{G}$, of a residually finite group $G$. We briefly recall its definition.

Fix a generating family $S$ of $G$. Consider the function $\rho_{S}$, that to a natural number $n$ associates the smallest number $k$ such that, for any non-trivial element of length at most $n$ in $G$, there exists a finite quotient of $G$ of order at most $k$, such that the image of this element in that quotient is non-trivial. This is the depth function of $G$ with respect to the generating family $S$.

For two functions $f$ and $g$ defined on natural numbers, note $f \succeq g$ to mean that there exists a constant $C$ such that for any number $n, f(n) \geq C g(C n)$. It is easy to see that one defines an equivalence relation $\simeq$ by putting $f \simeq g$ if and only if $f \succeq g$ and $g \succeq f$.

If $S$ and $S^{\prime}$ are two different generating families of a group $G$, the functions $\rho_{S}$ and $\rho_{S^{\prime}}$ will satisfy $\rho_{S} \simeq \rho_{S^{\prime}}([3])$. Thus one can define uniquely the depth function $\rho_{G}$ of the group $G$ by considering the equivalence class for $\simeq$ of a function $\rho_{S}$, for some generating family $S$.

The interaction between the depth function of a group and it having CFQ makes it worth mentioning here, and in fact, an ancestor of the depth function can be found in McKinsey's original article, [19, where an upper bound for what would be a "depth function" for lattices (partially ordered sets) is computed. This interaction also appears in 4 .

Consider a residually finite group $G$, that has CFQ, generated by a family $S$. We know that $G$ is then co-re, because McKinsey's algorithm applies: list all quotients of $G$ in order, and check wether an element has non-trivial image in one of those quotients. How long this will take is bounded by the depth function. In particular, if for an element $w$ of length $n$ of $G$, the algorithm has already tested all quotients of size at most $\rho_{S}(n)$, and not found a quotient in which $w$ is non-trivial, then $w=e$.

Proposition 23. Let $G$ be a finitely generated residually finite group with $C F Q$.

If there exists a generating family $S$ of $G$ and a recursive function $h$ that satisfies $\rho_{S} \leq h$, then $G$ has solvable word problem.

If $G$ has solvable word problem, then for any generating family $S$ of $G$, the depth function of $G$ with respect to $S$ is recursive.

Proof. The first claim follows from the sentence that immediately precedes this proposition. The second claim is straightforward.

Remark 24. The depth function $\rho_{G}$ of $G$ is defined up to the equivalence relation $\simeq$. In the statement of this last proposition appears the condition "there exists a recursive function $h$ that satisfies $\rho_{S} \leq h$ ". If two functions $f$ and $g$ satisfy $f \simeq g$, and if there exists a recursive function $h$ that satisfies $f \leq h$, then of course there exists a recursive function $h^{\prime}$ that satisfies $g \leq h^{\prime}$, and thus it makes sense to say that there exists a recursive function that bounds the depth function $\rho_{G}$. Note however that it is possible for a recursive function $f$ to be equivalent, for $\simeq$, to a non-recursive function $g$, (and even worse: the equivalence class for $\simeq$ of any recursive function contains a non-recursive function), and because of this, the term 
"recursive depth function", must be manipulated carefully. In what follows, we will say that a group $G$ has recursive depth function if for any generating family $S$ of the group $G$, the function $\rho_{S}$ is recursive. Proposition 23 implies that for a group with CFQ, either all its relative depth functions $\rho_{S}$ are recursive, or none of them are. From this it is natural to ask the following problem:

Problem 25. Let $G$ be a finitely generated residually finite group, and $S$ and $S^{\prime}$ two generating families of $G$. Is it possible that exactly one of $\rho_{S}$ and $\rho_{S^{\prime}}$ be recursive?

The following follows immediately from Proposition 23.

Corollary 26. For residually finite groups with CFQ, having solvable word problem is equivalent to having recursive depth function.

Theorem 3 thus asserts the existence of a group with CFQ but non-recursive depth function. Note that result similar to this corollary exists, that relates the Dehn function of a finitely presented group and solvability of the word problem in it.

In [14], it was shown that for any recursive function $f$, there is a finitely presented residually finite group with depth function greater than $f$, and yet with word problem solvable in polynomial time. For such a group, McKinsey's algorithm is far from being optimal. The group constructed in order to prove Theorem 2 shows that, for non-finitely presented groups, the situation can be even more extreme: in it, the word problem is solvable, but not by McKinsey's algorithm.

It was explained in the first section of this paper that conjugacy separability and subgroup separability, when confronted to the property of having computable finite quotients, play a role similar to residual finiteness, but with respect to the conjugacy problem and the generalized word problem. It is then natural to introduce functions similar to the depth function of residually finite groups, but that quantify conjugacy separability and subgroup separability. This was first done in [15] for conjugacy separability, and in [6] for subgroup separability. What was said in this section about recursiveness of the depth function translates easily to those two functions.

\section{Examples of Groups With CFQ}

In this section, we give examples of infinitely presented groups that have CFQ. Those examples contain some commonly used infinitely presented groups.

4.1. Wreath products. The (restricted) wreath product of two groups $G$ and $H$, noted $H \nmid G$, is the semi direct product $H \ltimes \bigoplus_{H} G$, where $H$ acts on $\bigoplus_{H} G$ by permuting the indices. The wreath product $H \imath G$ of two finitely generated groups is always finitely generated, however, by a theorem of Baumslag ([2]), it is finitely presented only if $G$ is finitely presented and $H$ is finite (excluding the case where $G$ is the trivial group). If $\left\langle S_{G} \mid R_{G}\right\rangle$ and $\left\langle S_{H} \mid R_{H}\right\rangle$ are presentations respectively of $G$ and $H$, with $S_{G} \cap S_{H}=\varnothing$, then a presentation of $H \prec G$ is given by the following:

$$
\left\langle S_{G}, S_{H} \mid R_{G}, R_{H},\left[h g_{1} h^{-1}, g_{2}\right],\left(g_{1}, g_{2}, h\right) \in S_{G} \times S_{G} \times(H \backslash\{1\})\right\rangle
$$

(where by $h \in H \backslash\{1\}$ we actually mean "for each $h \in H \backslash\{1\}$, choosing a way of expressing $h$ in terms of a product of the generators of $S_{H}$ ").

The following proposition gives necessary and sufficient conditions for a wreath product to have CFQ. 
Proposition 27. The wreath product $H \frown G$ of two finitely generated groups $G$ and $H$ has $C F Q$ if and only if either $H$ has $C F Q$ and is infinite, or if $H$ is finite and $G$ has $C F Q$.

Proof. Suppose that $H \imath G$ has CFQ. From the presentation of $H \imath G$ given above, it is easy to see that $H$ can be obtained as a quotient of $H \prec G$ by adding only finitely many relations to it. Thus by Proposition 20, $H$ has CFQ.

Suppose additionally that $H$ is finite. A finite group $F$, marked by a function $f$, is a quotient of $G$ if and only if the function $g$, defined as the identity on the generators of $H$ and as $f$ on those of $G$, which thus sends a generating family of $H$ 乙 $G$ to one of $H$ 乙 F , can be extended to a morphism. But because $H$ is finite, $H \imath F$ is also finite, and thus it can be determined whether or not it is a quotient of $H$ 乙 $G$. This proves that $G$ has CFQ.

For the converse, suppose first that $H$ is finite and that $G$ has CFQ. Because $H$ is finite, a presentation for $H \imath G$ can be obtained by adding finitely many relations to a presentation of $G * H$, which has CFQ because both $G$ and $H$ have. Thus Proposition 20 applies again.

Suppose now that $H$ is infinite and has CFQ. In a finite quotient of $H \prec G$, some non-trivial element of $H$ must necessarily have a trivial image. But it is easy to see, from the presentation of $H$ 乙 $G$ given above, that, for any element $h \neq 1$ of $H$, the relation $h=1$ together with the relations of $H$ \G will always also imply that $\left[g_{1}, g_{2}\right]=1$, for any pair $\left(g_{1}, g_{2}\right)$ of elements of $G$. This implies that any finite quotient of $H \backslash G$ is in fact a finite quotient of $H \backslash G_{a b}$, where $G_{a b}$ denotes the abelianization $G /[G, G]$ of $G$. It thus suffices to prove that if $H$ has CFQ and $G$ is abelian, then the wreath product $H$ ¿ $G$ also has CFQ.

Consider a finite group $F$ together with a function $f$ that goes from a generating family of $H$ ₹ $G$ to $F$. To decide whether $f$ can be extended to a morphism, one can first check whether the restriction $f_{H}$ of $f$ to a generating family of $H$ extends as a morphism from $H$ onto a subgroup $F_{H}$ of $F$, using the fact that $H$ has CFQ. If it does not, then $f$ cannot be extended to a group morphism. If it does, one can compute a presentation of $F_{H} . \quad F$ is then a quotient of $H<G$ if and only if it is a quotient of $F_{H}$ 乙 $G$. But $G$ is finitely presented, being abelian, and $F_{H}$ is finite, thus this wreath product is finitely presented, and a finite presentation of it can be obtained from the presentation of $F_{H}$. This presentation can in turn be used to decide whether $F$ is a quotient of $F_{H} \imath G$, and thus of $H \imath G$.

4.2. L-presented groups. L-presented groups were introduced in 1]. An Lpresentation, or finite endomorphic presentation, is a quadruple $\left\langle S\left|R_{1}\right| \Phi \mid R_{2}\right\rangle$, where $S$ is a finite set of generating symbols, $R_{1}$ and $R_{2}$ are finite sets of relations, i.e. of elements of the free group $\mathcal{F}_{S}$ defined over $S$, and $\Phi$ is a finite set of endomorphisms of $\mathcal{F}_{S}$. Such a presentation defines a presentation, in the usual sense of the term, by adding to $R_{1} \cup R_{2}$, as further relations, all elements of $\mathcal{F}_{S}$ that can be obtained from $R_{2}$ by iterating the endomorphisms of $\Phi$. (This process can be carried out effectively, an L-presentation is thus always a recursively enumerable presentation.)

It was proven in [13, that a coset enumeration process can be carried out in L-presented groups, and that the membership problem for finite index subgroups is solvable in L-presented groups. This, we have seen, directly implies that those groups have CFQ.

Proposition 28. L-presented groups have CFQ. 
Many groups of interest that are not finitely presented were proven to admit Lpresentations, including groups of intermediate growth, and various groups acting on rooted trees, like the Gupta-Sidki group (see [1]).

Note that many of those groups were proven to be conjugacy separable ([24]) or subgroup separable ([10, 9]). The fact that they have CFQ thus completes for those groups the proof of solvability of the conjugacy problem, or of the generalized word problem. (Although in some cases, solutions to these problems are known, that do not rely on McKinsey's algorithm).

\section{MAin Unsolvability RESUlts}

In this section, we prove the two main theorems of this paper.

Theorem 3 follows from the construction that Slobodskoi used in 23 to prove undecidability of the universal theory of finite groups. However, the exact result we use was implicit in [23, and it was first pointed out to hold by Bridson and Wilton in [5]. We first show how, thanks to this result, the proof of Theorem 3 is immediate.

We then detail Dyson's construction from [8], which, with [20, contained the first examples of re residually finite groups without solvable word problem. We show that this construction provides us with another proof of Theorem 3, which has the advantage of relying on groups whose structure is very explicit. We finally use Dyson's construction to build a residually finite group with solvable word problem, and yet without CFQ, thus proving Theorem 2 ,

5.1. Slobodskoi's example for Theorem 3. Recall that the finitary image $G_{f}$ of a group $G$ is its biggest residually finite quotient.

To each finite presentation, one can associate a residually finite group, by taking the finitary image of the group defined by this presentation. If $\pi$ is a finite presentation for a group $G$, and if $G_{f}$ is the finitary image of $G$, say that $\pi$ is a finite presentation of $G_{f}$ as a residually finite group, and that $G_{f}$ is finitely presentable as a residually finite group.

In 1981, in [23, Slobodskoi proved that the universal theory of finite groups is undecidable. As a consequence of his paper, groups that are finitely presented as residually finite groups do not have uniformly solvable word problem. But in fact, more can be deduced from the proof that appears in [23], as was made explicit by Bridson and Wilton in [5]:

Theorem 29 (5, Theorem 2.1). There exists a finitely presented group $G$ in which there is no algorithm to decide which elements have trivial image in every finite quotient.

Two other equivalent formulations of this theorem are the following ones:

Theorem. There exists a finitely presented group $G$ whose finitary image is not recursively presented.

Theorem. There exists a group $H$ which is finitely presentable as a residually finite group, but which has unsolvable word problem.

This theorem then directly implies Theorem 3 .

Proof of Theorem [3. A group which is finitely presented as a residually finite group must have CFQ, by Proposition 22. Thus if such a group has unsolvable word 
problem, it satisfies the criteria required by Theorem 3. A group, finitely presented as a residually finite group, with unsolvable word problem exists by Theorem 29.

5.2. Dyson's Groups. These groups are amalgamated products of two lamplighter groups.

The lamplighter group $L$ is the wreath product $\mathbb{Z} \imath \mathbb{Z} / 2 \mathbb{Z}$ of $\mathbb{Z}$ and of the order two group. It admits the following presentation:

$$
\left\langle a, \varepsilon \mid \varepsilon^{2},\left[\varepsilon, a^{i} \varepsilon a^{-i}\right], i \in \mathbb{Z}\right\rangle
$$

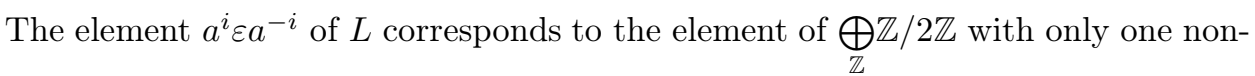
zero coordinate in position $i \in \mathbb{Z}$. We call it $u_{i}$. Consider another copy $\hat{L}$ of the lamplighter group, together with an isomorphism from $L$ to $\hat{L}$ we note $g \mapsto \hat{g}$. For each subset $\mathcal{A}$ of $\mathbb{Z}$, define $L(\mathcal{A})$ to be the amalgamated product of $L$ and $\hat{L}$, with $u_{i}=a^{i} \varepsilon a^{-i}$ identified with $\hat{u}_{i}=\hat{a}^{i} \hat{\varepsilon} \hat{a}^{-i}$ for each $i$ in $\mathcal{A}$. It has the following presentation:

$$
\left\langle a, \hat{a}, \varepsilon, \hat{\varepsilon} \mid \varepsilon^{2}, \hat{\varepsilon}^{2},\left[\varepsilon, a^{i} \varepsilon a^{-i}\right],\left[\hat{\varepsilon}, \hat{a}^{i} \hat{\varepsilon} \hat{a}^{-i}\right], i \in \mathbb{Z}, a^{j} \varepsilon a^{-j}=\hat{a}^{j} \hat{\varepsilon} \hat{a}^{-j}, j \in \mathcal{A}\right\rangle
$$

Recall that the double of a group $G$ over a subgroup $H$ is the free product of two copies of $G$ amalgamated along the identity over $H$, denote it $G *_{H} G$. Dyson's group associated to $\mathcal{A}$ is thus the double of the Lamplighter group $L$ over the subgroup generated by elements of the form $a^{i} \varepsilon a^{-i}$, for $i$ in $\mathcal{A}$. Call $H_{\mathcal{A}}$ this subgroup.

For $n$ a non-zero natural number, denote $L(\mathcal{A})_{n}$ the group $\left\langle L(\mathcal{A}) \mid a^{n}, \hat{a}^{n}\right\rangle$.

Call $\mathcal{A} \bmod n$ the set $\{r \in\{0, \ldots, n-1\}, \exists a \in \mathcal{A}, a \equiv \operatorname{rmod} n\}$.

Lemma 30. $L(\mathcal{A})_{n}$ is finitely presented and residually finite. It is the amalgamated product of two copies of the finite wreath product $\mathbb{Z} / n \mathbb{Z} \imath \mathbb{Z} / 2 \mathbb{Z}$, and it admits the following presentation:

$$
\begin{array}{r}
\langle a, \hat{a}, \varepsilon, \hat{\varepsilon}| a^{n}, \hat{a}^{n}, \varepsilon^{2}, \hat{\varepsilon}^{2},\left[\varepsilon, a^{i} \varepsilon a^{-i}\right],\left[\hat{\varepsilon}, \hat{a}^{i} \hat{\varepsilon} \hat{a}^{-i}\right], 0 \leq i \leq n-1, \\
\left.a^{j} \varepsilon a^{-j}=\hat{a}^{j} \hat{\varepsilon} \hat{a}^{-j}, j \in \mathcal{A} \bmod n\right\rangle
\end{array}
$$

Proof. The given presentation is obtained from the presentation of $L(\mathcal{A})$, adding relations $a^{n}$ and $\hat{a}^{n}$, and simplifying the relations as can be done. It then follows from that presentation that $L(\mathcal{A})_{n}$ is an amalgamated product of two copies of the finite wreath product $\mathbb{Z} / n \mathbb{Z} \imath \mathbb{Z} / 2 \mathbb{Z}$. Finally it is well known that an amalgamated product of finite groups is residually finite (see [17]).

The properties of the group $L(\mathcal{A})$ are stated using the profinite topology on $\mathbb{Z}$. A basis of open sets for $\mathcal{P} \mathcal{T}(\mathbb{Z})$ consists in sets of the form $n+p \mathbb{Z}$, for $n$ and $p$ integers. Thus a subset $\mathcal{A}$ of $\mathbb{Z}$ is open in $\mathcal{P} \mathcal{T}(\mathbb{Z})$ if and only if for every $n$ in $\mathcal{A}$ there exists an integer $p$ such that $n+p \mathbb{Z} \subseteq \mathcal{A}$.

We can now state the properties of the group $L(\mathcal{A})$ that are relevant to this work.

Proposition 31. Let $\mathcal{A}$ be a subset of $\mathbb{Z}$.

(1) $L(\mathcal{A})$ is re, co-re or has solvable word problem if and only if $\mathcal{A}$ is respectively re, co-re or recursive.

(2) $L(\mathcal{A})$ is residually finite if and only if $\mathcal{A}$ is closed in $\mathcal{P} \mathcal{T}(\mathbb{Z})$. 
(3) $L(\mathcal{A})$ has $C F Q$ if and only if the function which to $n$ associates $\mathcal{A} \bmod n$ is recursive.

The first two points of this proposition were proven in [8]. We still include proofs for those two statements. The proof given for (1) is exactly that of Dyson. We then give two proofs of (2), one shorter and more conceptual than that of [8], which was suggested to us by the anonymous referee, and another one which, although not as clear as the previous one, can readily be rendered effective, and in the proof of Proposition 34 we refer ourselves to this second proof.

Notice finally that the condition which appears in (3) could be stated as: $\mathcal{A}$ is determinable in finite quotients of $\mathbb{Z}$ (see Definition 5 ).

Proof. We prove all three points in order.

If $L(\mathcal{A})$ is re or co-re, then clearly so is $\mathcal{A}$, as $n$ belongs to $\mathcal{A}$ if and only if $u_{n}=\hat{u}_{n}$ in $L(\mathcal{A})$, which proves one direction of $(1)$.

It is clear that if $\mathcal{A}$ is re, the presentation of $L(\mathcal{A})$ given above is re as well.

Suppose now that $\mathcal{A}$ is co-re. We can enumerate the complement of $\mathcal{A}$, and thus enumerate elements of the form:

$$
\text { (*) } w=a^{\alpha_{1}} x_{1} \hat{a}^{\beta_{1}} y_{1} \ldots a^{\alpha_{k}} x_{k} \hat{a}^{\beta_{k}} y_{k} z
$$

where $\alpha_{i}, \beta_{i} \in \mathbb{Z}, x_{1}, \ldots, x_{k}$ and $y_{1}, \ldots, y_{k}$ are elements of the base groups of $L$ and $\hat{L}$ that have null components corresponding to indices in $\mathcal{A}$, and $z$ is any element in $L$ or in $\hat{L}$. The elements written this way are exactly the elements in normal form for the amalgamated product $L(\mathcal{A})$. Recall that the normal form in an amalgamated product ([16]) necessitates two choices of transversals, here one for $L /\left(\bigoplus_{\mathcal{A}} \mathbb{Z} / 2 \mathbb{Z}\right)$ and one for $\hat{L} /\left(\bigoplus_{\mathcal{A}} \mathbb{Z} / 2 \mathbb{Z}\right)$, and that an element in normal form is a consecutive product of an element of one transversal, followed by one of the other, etc, terminated by any element of one of these groups. But elements $a^{\alpha} x$, with $\alpha$ in $\mathbb{Z}$ and $x$ in the base group with null components on $\mathcal{A}$, indeed form the most natural transversal for $L /\left(\bigoplus_{\mathcal{Z}} \mathbb{Z} / 2 \mathbb{Z}\right)$. Thus any non-trivial element $g$ of $L(\mathcal{A})$ is equal to exactly one element in this enumeration. Ideally, we would then enumerate words that give the identity in $L(\mathcal{A})$, and listing words that can be obtained concatenating a word in normal form to a word that defines the identity would give the desired enumeration. Since $L(\mathcal{A})$ is not supposed re, we cannot directly enumerate this set of trivial words, but we will over-approximate it by a re set. For $w$ as in (*), note $B_{w}$ the set of all indices that appear in elements $x_{i}$ or $y_{i}$ of the base groups ( $B_{w}$ can be empty). The over approximation consists of the words corresponding to the identity in $L\left(\mathbb{Z} \backslash B_{w}\right)$. Note that $w$ is also in normal form in $L\left(\mathbb{Z} \backslash B_{w}\right)$, thus non-trivial there. Of course, $B_{w}$ is finite, thus $\mathbb{Z} \backslash B_{w}$ is re, thus as we already remarked, we can enumerate words (in $a, \hat{a}, \varepsilon, \hat{\varepsilon}$ ) that correspond to the identity in $L\left(\mathbb{Z} \backslash B_{w}\right)$. Then for any such word $w_{1}$, the product $w w_{1}$ corresponds to a non-identity element of $L\left(\mathbb{Z} \backslash B_{w}\right)$, thus to a non-identity element in $L(\mathcal{A})$, since, as $\mathcal{A} \subseteq\left(\mathbb{Z} \backslash B_{w}\right), L\left(\mathbb{Z} \backslash B_{w}\right)$ satisfies more relations than $L(\mathcal{A})$. Thus enumerating products $w w_{1}$ with $w_{1}=e$ in $L\left(\mathbb{Z} \backslash B_{w}\right)$ will only yield non-identity elements in $L(\mathcal{A})$. What's more, every element of the form $w w_{2}$, where $w_{2}$ is a word that is the identity in $L(\mathcal{A})$, will arise this way, again because $L\left(\mathbb{Z} \backslash B_{w}\right)$ satisfies more relations than $L(\mathcal{A})$.

Because the algorithm that enumerates relations in $L\left(\mathbb{Z} \backslash B_{w}\right)$ depends recursively on $w$, this process can be applied simultaneously to all words $w$ in normal form, 
giving an enumeration of all words that correspond to non-identity elements of $L(\mathcal{A})$, thus proving that $L(\mathcal{A})$ is co-re.

Finally $\mathcal{A}$ is recursive if and only if it is both re and co-re, if and only if $L(\mathcal{A})$ has solvable word problem.

This ends the proof of (1).

We first sketch a conceptually simple proof of (2).

Recall that the group $L(\mathcal{A})$ is the double of the lamplighter group $L$ over the subgroup $H_{\mathcal{A}}$ generated by elements of the form $a^{i} \varepsilon a^{-i}$, for $i$ in $\mathcal{A}$. The statement (2) is implied by the two following arguments:

- the double of a residually finite group $G$ over a subgroup $H$ is itself residually finite if and only if $H$ is separable in $G$;

- the subgroup $H_{\mathcal{A}}$ is separable in $L$ if and only if $\mathcal{A}$ is separable in $\mathbb{Z}$.

Those arguments are easy to check, and because a second proof for (2) follows, we do not detail them.

We now give another proof of (2), which can be easily rendered effective. We in fact prove slightly more than (2): for a subset $\mathcal{A}$ of $\mathbb{Z}$, the finitary image $L(\mathcal{A})_{f}$ of $L(\mathcal{A})$ is $L(\overline{\mathcal{A}})$, where $\overline{\mathcal{A}}$ denotes the closure of $\mathcal{A}$ in $\mathcal{P} \mathcal{T}(\mathbb{Z})$. First we show that if $n$ belongs to $\overline{\mathcal{A}}$, then in any finite quotient $(F, f)$ of $L(\mathcal{A})$, the images of $u_{n}$ and $\hat{u}_{n}$ are the same in $F$. Let $(F, f)$ be some finite quotient of $L(\mathcal{A})$. Call $p$ and $p^{\prime}$ the orders of $f(a)$ and $f(\hat{a})$ in $F$. Then, because $n$ belongs to $\overline{\mathcal{A}}, n+p p^{\prime} \mathbb{Z}$ must meet with $\mathcal{A}$, as it is a neighborhood of $n$. Thus we have $k$ such that $n+p p^{\prime} k \in \mathcal{A}$, that is, such that $u_{n+p p^{\prime} k}=\hat{u}_{n+p p^{\prime} k}$ in $L(\mathcal{A})$. Then, in $F$ (we omit to write the homomorphism onto $F$ ):

$$
\begin{aligned}
u_{n} & =\left(a^{p}\right)^{p^{\prime} k} u_{n}\left(a^{p}\right)^{-p^{\prime} k}=a^{p p^{\prime} k} u_{n} a^{-p p^{\prime} k} \\
& =u_{n+p p^{\prime} k} \\
& =\hat{u}_{n+p p^{\prime} k} \\
& =\left(\hat{a}^{p^{\prime}}\right)^{p k} \hat{u}_{n}\left(\hat{a}^{p^{\prime}}\right)^{-p k}=\hat{u}_{n}
\end{aligned}
$$

This shows that $L(\mathcal{A})_{f}$ is a quotient of $L(\overline{\mathcal{A}})$. It is then sufficient to see that $L(\overline{\mathcal{A}})$ is residually finite to see that $L(\mathcal{A})_{f}=L(\overline{\mathcal{A}})$.

We suppose that $\mathcal{A}$ is closed to omit the closure notation. Let $w$ be a non-identity element of $L(\mathcal{A})$, and write it in normal form $w=a^{\alpha_{1}} x_{1} \hat{a}^{\beta_{1}} y_{1} \ldots a^{\alpha_{k}} x_{k} \hat{a}^{\beta_{k}} y_{k} z$, as in the proof of (1).

Suppose first that the normal form is the trivial one: $w=z$ with $z$ in $L$ or in $\hat{L}$. Then $w$ is non-trivial in the quotient of $L(\mathcal{A})$ obtained by identifying the two copies $L$ and $\hat{L}$ of the lamplighter group (i.e. $\langle L(\mathcal{A}) \mid a=\hat{a}, \varepsilon=\hat{\varepsilon}\rangle$ ), which is just the lamplighter group itself, which is residually finite.

We can now suppose the normal form has several terms. Each $x_{i}$ is an element of $\bigoplus \mathbb{Z} / 2 \mathbb{Z}$, that is to say a product $\prod u_{k_{i, j}}$ with $k_{i, j} \notin \mathcal{A}$. Because $\mathcal{A}$ is closed, $\mathbb{Z} \backslash \mathcal{A}$

for each such $k_{i, j}$ there is $p_{i, j}$ that satisfies $\left(k_{i, j}+p_{i, j} \mathbb{Z}\right) \cap \mathcal{A}=\varnothing$. Similarly, for each $\hat{x}_{i}$, introduce integers $p_{i, j}^{\prime}, j=1,2, \ldots$. Call $N$ the product $\prod p_{i, j} \prod p_{i, j}^{\prime}$. (It is 1 if, for all $i, x_{i}=\hat{x}_{i}=e$.) We claim that $w$ is non-trivial in $L(\mathcal{A})_{N}$. Indeed, $N$ was chosen so that for any $(i, j), k_{i, j}$ (or its remainder modulo $N$ ) does not belong to $\mathcal{A} \bmod N$. This implies that $w$ is also in normal form in $L(\mathcal{A})_{N}$ (by Lemma 30), and thus non-trivial there. 
Again by Lemma 30, $L(\mathcal{A})_{N}$ is residually finite, so we've proven that $L(\mathcal{A})$ is residually residually finite, which of course is the same as residually finite.

Finally we prove (3).

Suppose $\mathcal{A} \bmod n$ depends recursively of $n$. Let $(F, f)$ be a finite group together with a function $f$ from $\{a, \hat{a}, \varepsilon, \hat{\varepsilon}\}$ to $F$. To determine whether $f$ defines a homomorphism, compute the orders of $f(a)$ and of $f(\hat{a})$, and let $n$ be their product. If $f$ extends to a morphism, this morphism factors through the projection $\pi: L(\mathcal{A}) \rightarrow L(\mathcal{A})_{n}$. By Lemma 30, a finite presentation for $L(\mathcal{A})_{n}$ can be found from the computation of $\mathcal{A} \bmod n$. It can then be determined in finite time from this presentation whether $f$ defines a homomorphism from $L(\mathcal{A})_{n}$ to $F$.

Suppose now that $L(\mathcal{A})$ has $\mathrm{CFQ}$. Let $n$ be a natural number. To compute $\mathcal{A} \bmod n$, consider all possible presentations for $L(\mathcal{A})_{n}$ : for $B \subset\{0, \ldots, n-1\}$, define the presentation $\prod_{B}$ :

$$
\begin{array}{r}
\langle a, \hat{a}, \varepsilon, \hat{\varepsilon}| a^{n}, \hat{a}^{n}, \varepsilon^{2}, \hat{\varepsilon}^{2},\left[\varepsilon, a^{i} \varepsilon a^{-i}\right],\left[\hat{\varepsilon}, \hat{a}^{i} \hat{\varepsilon} \hat{a}^{-i}\right], 0 \leq i \leq n-1, \\
\left.a^{j} \varepsilon a^{-j}=\hat{a}^{j} \hat{\varepsilon} \hat{a}^{-j}, j \in B\right\rangle
\end{array}
$$

All these presentations define residually finite groups, and because they are finitely presented, they have CFQ. $L(\mathcal{A})_{n}$ also has CFQ, because it is obtained from $L(\mathcal{A})$ by adding two relations, thus we can start to enumerate the quotients of $L(\mathcal{A})_{n}$. Also start enumerating the quotients of all groups given by the presentations $\prod_{B}$, for $B \subset$ $\{0, \ldots, n-1\}$. Those $2^{n}$ lists are all different (because, as the presentations $\prod_{B}$ give residually finite groups, a list contains a finite group in which the images of $a^{j} \varepsilon a^{-j}$ and $\hat{a}^{j} \hat{\varepsilon}^{-j}$ differ if and only if $j$ does not belong to $B$ ), and only one corresponds to the list of quotients of $L(\mathcal{A})_{n}$. It can be determined, in a finite number of steps, which of those lists corresponds to $L(\mathcal{A})_{n}$, and thus which presentation $\prod_{B}$ gives a presentation of $L(\mathcal{A})_{n}$, and then one can conclude that $B=\mathcal{A} \bmod n$.

From Proposition 31, to prove Theorem 2, it suffices to build $\mathcal{A}$ with the following properties: $\mathcal{A}$ is closed in $\mathcal{P} \mathcal{T}(\mathbb{Z}), \mathcal{A}$ is recursive, there is no algorithm that takes $n$ as input and computes $\mathcal{A} \bmod n$. Similarly, to prove Theorem 3 , it suffices to build $\mathcal{A}$ such that: $\mathcal{A}$ is closed in $\mathcal{P} \mathcal{T}(\mathbb{Z}), \mathcal{A}$ is not recursive, but there is an algorithm that, given $n$ as input, computes $\mathcal{A} \bmod n$.

5.3. Building subsets of $\mathbb{Z}$ with prescribed properties. We first give an alternative proof of Theorem 3, before completing the proof of Theorem 2,

Lemma 32. There exists a non-recursive subset $\mathcal{A}$ of $\mathbb{Z}$, closed in $\mathcal{P} \mathcal{T}(\mathbb{Z})$, for which $\mathcal{A}$ modn depends recursively of $n$.

Note that without the closeness assumption, this result would be a lot easier: it is precisely the result of Proposition 7 , whose proof is very short, and yields a set that can be neither re nor co-re. However, for a closed set $\mathcal{A}$, the computation of $\mathcal{A} \bmod n$ will yield an enumeration of the complement of $\mathcal{A}$ : indeed, if $a$ is not in $\mathcal{A}$, some open set $a+b \mathbb{Z}$ must not meet $\mathcal{A}$, and thus $a$ is not in $\mathcal{A}$ mod $b$. This proves that if $\mathcal{A}$ is closed, and if $\mathcal{A} \bmod n$ is computable, then $\mathcal{A}$ is co-re. This is just the translation for Dyson's groups of: if $G$ is residually finite, and has CFQ, then $G$ is co-re.

Proof. We construct a set $\mathcal{B}$, which will be the complement of the announced $\mathcal{A}$. Thus it has to be open, re but not co-re, and for any $a$ and $b$, the question "is $a+b \mathbb{Z}$ a subset of $\mathcal{B}^{\prime \prime}$ has to be solvable in a finite number of steps. Indeed, $a$ belongs to 
$\mathcal{A} \bmod b$ if and only if $a+b \mathbb{Z}$ meets $\mathcal{A}$, if and only if $a+b \mathbb{Z}$ is not a subset of the complement of $\mathcal{A}$.

Call $p_{n}$ the $n$-th prime number. Define two sequences $\left(x_{n}\right)_{n \geq 0}$ and $\left(y_{n}\right)_{n \geq 1}$ by the following:

$$
\begin{aligned}
& x_{0}=1 \\
& x_{n}=p_{n} x_{n-1}^{2} \\
& y_{n}=x_{n-1}
\end{aligned}
$$

These sequences have the following properties:

- for any $n, x_{n} \mid x_{n+1}$ and $y_{n} \mid y_{n+1}$.

- for any integer $b$, there is some (computable) $n$ such that $b \mid x_{n}$ and $b \mid y_{n}$.

- $p_{k}$ divides $x_{n}$ if and only if $k \geq n$, and $p_{k}$ divides $y_{n}$ if and only if $k>n$.

- for integers $k, k^{\prime}, n, n^{\prime}$, with $k \leq n$ and $k^{\prime} \leq n^{\prime}, y_{k}+x_{n} \mathbb{Z}$ and $y_{k^{\prime}}+x_{n^{\prime}} \mathbb{Z}$ are disjoint if and only if $k \neq k^{\prime}$, and otherwise one is a subset of the other.

All these are clear, the fourth point follows from the third, by remarking that elements of $y_{k}+x_{n} \mathbb{Z}$ are all multiples of $p_{0}, p_{1}, \ldots p_{k-1}$, but none of them is a multiple of $p_{k}$.

Consider a recursive function $f$ whose image is re but not co-re. Assume that for any $n, 1 \leq f(n) \leq n$ (it is easy to see that such a function exists). Then we define $\mathcal{B}$ as the union:

$$
\mathcal{B}=\bigcup_{n \in \mathbb{N}^{*}} y_{f(n)}+x_{n} \mathbb{Z}
$$

Since $f$ is a recursive function, $\mathcal{B}$ is re. It is not co-re, however, because $y_{m}$ belongs to $\mathcal{B}$ if and only if $m$ belongs to the image of $f$ (this follows directly from the properties of the sequences $\left(x_{n}\right)_{n \geq 0}$ and $\left.\left(y_{n}\right)_{n \geq 1}\right)$.

$\mathcal{B}$ is an open set, because it is defined as an union of open sets.

All that is left to see is that we can decide, for $a$ and $b$ integers, whether $a+b \mathbb{Z}$ is a subset of $\mathcal{B}$. Suppose that $a<b$. If $a=0$, then $0 \in a+b \mathbb{Z}$, but $0 \notin \mathcal{B}$, thus $a+b \mathbb{Z}$ is not a subset of $\mathcal{B}$. If $a$ is non-zero, no element of $a+b \mathbb{Z}$ is divisible by $b$. Thus, because of the second property of the sequences $\left(x_{n}\right)_{n \geq 0}$ and $\left(y_{n}\right)_{n \geq 1}$ quoted above, there exists $N$ such that if $N \leq k \leq n$, then $a+b \mathbb{Z} \cap y_{k}+x_{n} \mathbb{Z}=\varnothing$. Thus $a+b \mathbb{Z}$ is a subset of $\mathcal{B}$ if and only if it is a subset of the set $\mathcal{B}_{N}$, defined by:

$$
\mathcal{B}_{N}=\bigcup_{n \in \mathbb{N}^{*}, f(n) \leq N} y_{f(n)}+x_{n} \mathbb{Z}
$$

Define a pseudo-inverse $g$ of $f$ by $g(m)=\inf \{n, f(n)=m\}$. Because we chose $f$ such that for any $n, f(n) \leq n$, for any $m, g(m) \geq m$. If $m$ is not in the image of $f$, put $g(m)=\infty$. The set $\mathcal{B}_{N}$ can be expressed as the disjoint union:

$$
\mathcal{B}_{N}=\bigcup_{k \in \operatorname{Im}(f), k \leq N} y_{k}+x_{g(k)} \mathbb{Z}
$$

Because $x_{k} \mid x_{g(k)}, \mathcal{B}_{N}$ is contained in the set $\mathcal{C}_{N}$, defined by:

$$
\mathcal{C}_{N}=\bigcup_{k \leq N} y_{k}+x_{k} \mathbb{Z}
$$

It can be determined whether $a+b \mathbb{Z}$ is contained in $\mathcal{C}_{N}$, because the sequences $\left(x_{n}\right)_{n \geq 1}$ and $\left(y_{n}\right)_{n \geq 1}$ can be computed. If $a+b \mathbb{Z}$ is not contained in $\mathcal{C}_{N}$, then it is not contained in $\mathcal{B}_{N}$ either. 
If it is contained in $\mathcal{C}_{N}, a+b \mathbb{Z}$ is contained in $\mathcal{B}_{N}$ if and only if, for each $k$, $a+b \mathbb{Z} \cap y_{k}+x_{k} \mathbb{Z}$ is contained in $\mathcal{B}_{N}$. But, because $\mathcal{B}_{N}$ and $\mathcal{C}_{N}$ are disjoint unions, $a+b \mathbb{Z} \cap y_{k}+x_{k} \mathbb{Z}$ is contained in $\mathcal{B}_{N}$ if and only if it is contained in $y_{k}+x_{g(k)} \mathbb{Z}$. (If $k$ is not in $\operatorname{Im}(f), g(k)=\infty$, by convention $y_{k}+x_{g(k)} \mathbb{Z}=\left\{y_{k}\right\}$.) Now this question can be effectively answered. If $a+b \mathbb{Z} \cap y_{k}+x_{k} \mathbb{Z}$ is empty, there is nothing to do. Otherwise, it is of the form $t+\operatorname{lcm}\left(b, x_{k}\right) \mathbb{Z}$. Enumerate $f(1), f(2), \ldots, f\left(\operatorname{lcm}\left(b, x_{k}\right)\right)$. Either $k$ is in that list, in which case $g(k)$ can be computed and the question "is $a+b \mathbb{Z} \cap y_{k}+x_{k} \mathbb{Z}$ contained in $y_{k}+x_{g(k)} \mathbb{Z}$ " can be settled, or $k$ does not appear in the enumeration, which shows that $g(k)$ is greater than $\operatorname{lcm}\left(b, x_{k}\right)$. It this last case, as $x_{g(k)}$ is greater than $g(k), y_{k}+x_{g(k)} \mathbb{Z}$ cannot contain any set of the form $t+\operatorname{lcm}\left(b, x_{k}\right) \mathbb{Z}$.

This Lemma allows us to give another proof of Theorem 3 .

Proof of Theorem [3, alternate. It follows from Proposition 31 that the group $L(\mathcal{A})$, where $\mathcal{A}$ is the set constructed in Lemma 32, satisfies the requirements of Theorem 3.

This group has a depth function which cannot be smaller than a recursive function. We now prove the last lemma which ends the proof of Theorem 2 .

Lemma 33. There exists a recursive subset $\mathcal{A}$ of $\mathbb{Z}$, closed in $\mathcal{P} \mathcal{T}(\mathbb{Z})$, for which $\mathcal{A} \bmod n$ does not depend recursively of $n$.

Proof. Call $p_{n}$ the $n$-th prime number. Fix some effective enumeration $M_{1}, M_{2}, \ldots$ of all Turing machines. Consider the following process: start running simultaneously all those machines, as is done to show that the halting problem is re. While running calculations on the $n$-th machine, at each new step in the computation, produce a new power of $p_{2 n}: p_{2 n}, p_{2 n}^{2}, p_{2 n}^{3} \ldots$ If the computation on this machine stops after $k$ steps, end the list $p_{2 n}, p_{2 n}^{2}, \ldots, p_{2 n}^{k}$ already produced with $p_{2 n+1}^{k+1}$.

Call $\mathcal{A}$ the set of all powers of prime numbers obtained this way. $\mathcal{A}$ is obviously re, as it was defined by an effective enumeration process. It is even recursive. Indeed, for a number $x$, if $x$ is not the power of a prime, then $x$ is not in $\mathcal{A}$. If it is the power of a prime of even index, say $x=p_{2 n}^{k}$, then $x$ belongs to $\mathcal{A}$ if and only if the $n$-th Turing machine does not stop in less than $k$ calculations steps. This question can be effectively settled. Similarly, if $x$ is the power of a prime of odd index, $x=p_{2 n+1}^{k}$, then $x$ belongs to $\mathcal{A}$ if and only if the $n$-th Turing machine stops in exactly $k$ calculations steps, this also can be determined.

Of course, $\mathcal{A} \bmod m$ does not depend recursively of $m$. Indeed, the question: "does 0 belong to $\mathcal{A} \bmod p_{2 n+1}$ ?" is, by construction, equivalent to "does the $n$-th Turing machine halt?".

Finally, we show that $\mathcal{A}$ is a closed set, which is equivalent to finding, for any $x$ not in $\mathcal{A}$, a number $y$ such that $x+y \mathbb{Z}$ does not meet $\mathcal{A}$. If $x$ has several prime divisors, then $x+x \mathbb{Z}$ works, because any element of it has several prime divisors. If $x$ is the power of a prime of even index, $x=p_{2 n}^{k}$, and $x$ is not in $\mathcal{A}$, it must be that the $n$-th Turing machine stops in strictly less than $k$ steps. Thus the only elements in $\mathcal{A}$ that are multiples of $p_{2 n}$ will have a valuation in $p_{2 n}$ lower than $k$. Thus $x+x \mathbb{Z}$ will also work. The last case is if $x$ is the power of a prime of odd index, $x=p_{2 n+1}^{k}$. In this case, we claim that $x+p_{2 n+1} x \mathbb{Z}$ does not meet $\mathcal{A}$. Indeed, $x$ is the only power of $p_{2 n+1}$ contained in $x+p_{2 n+1} x \mathbb{Z}$, all other elements of it have at least two different prime divisors. 
With this we end the proof of Theorem 2 .

Proof of Theorem 圆. By Proposition 31, for a subset $\mathcal{A}$ of $\mathbb{Z}$, Dyson's group $L(\mathcal{A})$ satisfies the requirements of Theorem 2 provided that $\mathcal{A}$ is closed, recursive, and that the function $\mathcal{A} \bmod n$ is not computable. Lemma 33 provides such a set.

We finally remark that an upper bound to the depth function of the obtained group $L(\mathcal{A})$ can be effectively computed, and that the group obtained in Theorem 2 can be supposed to have recursive depth function.

Corollary 34. There exists a group with solvable word problem and recursive depth function, that does not have $C F Q$.

Proof. It appears clearly in the proof of Lemma 33 that the constructed $\mathcal{A}$ is effectively closed: if $x$ does not belong to it, then some $y$ such that $x+y \mathbb{Z}$ does not meet $\mathcal{A}$ can effectively be found. Going back to the proof of the first point of Proposition 31. it appears that, given a non-identity element $w$ of $L(\mathcal{A})$, the recursiveness of $\mathcal{A}$ permits to compute its normal form. Then, in the proof of the second point of that same proposition, it appears that, from this normal form and the effective closeness of $\mathcal{A}$, some integer $N$ can be effectively found, such that $w$ is non trivial in $L(\mathcal{A})_{N}$. A presentation of $L(\mathcal{A})_{N}$ cannot necessarily be found, but there are $2^{N}$ possible finite presentations for it, all of them with recursive depth function. Taking the supremum of those depth functions allows to compute a recursive upper bound to the depth function of $L(\mathcal{A})$.

Although it is not clear whether that depth function is recursive or not, by taking the direct product of the group $L(\mathcal{A})$ with a finitely presented group with recursive depth function greater that that of $L(\mathcal{A})$, which exists by [14], one obtains a group which still has solvable word problem and uncomputable finite quotients, and whose depth function is recursive.

In [22], we construct, also using Dyson's groups, a residually finite group $G$ with solvable word problem, that not only does not have CFQ, but that also is not effectively residually finite: there can be no algorithm that, given a non-trivial element $w$, gives a finite quotient $(F, f)$ in which the image of $w$ is non-trivial. This is done by constructing a closed subset $\mathcal{A}$ of $\mathbb{Z}$ that is not effectively closed.

\section{REFERENCES}

[1] L. Bartholdi. Endomorphic presentations of branch groups. Journal of Algebra, 268(2):419443 , oct 2003

[2] G. Baumslag. Wreath products and finitely presented groups. Mathematische Zeitschrift, 75(1):22-28, dec 1961.

[3] K. Bou-Rabee. Quantifying residual finiteness. Journal of Algebra, 323(3):729-737, feb 2010.

[4] K. Bou-Rabee and B. Seward. Arbitrarily large residual finiteness growth. Journal f ÃEr die reine und angewandte Mathematik (Crelles Journal), 2016(710), jan 2016.

[5] M. R. Bridson and H. Wilton. The triviality problem for profinite completions. Inventiones mathematicae, 202(2):839-874, feb 2015.

[6] J. Deré and M. Pengitore. Effective subgroup separability of finitely generated nilpotent groups. Journal of Algebra, 506:489-508, jul 2018.

[7] V. H. Dyson. The word problem and residually finite groups. American Mathematical Society Notices, 1964.

[8] V. H. Dyson. A family of groups with nice word problems. Journal of the Australian Mathematical Society, 17(4):414-425, jun 1974.

[9] A. Garrido. Abstract commensurability and the gupta-sidki group. Groups, Geometry, and Dynamics, 10(2):523-543, 2016 
[10] R. I. Grigorchuk and J. S. Wilson. A structural property concerning abstract commensurability of subgroups. Journal of the London Mathematical Society, 68(03):671-682, nov 2003.

[11] M. Hall. A topology for free groups and related groups. The Annals of Mathematics, 52(1):127, jul 1950.

[12] P. Hall. Finiteness conditions for soluble groups. Proceedings of the London Mathematical Society, s3-4(1):419-436, 1954.

[13] R. Hartung. Coset enumeration for certain infinitely presented groups. International Journal of Algebra and Computation, 21(08):1369-1380, dec 2011.

[14] O. Kharlampovich, A. Myasnikov, and M. Sapir. Algorithmically complex residually finite groups. Bull Math Sci, 7(2):309-352, mar 2017.

[15] S. Lawton, L. Louder, and D. McReynolds. Decision problems, complexity, traces, and representations. Groups, Geometry, and Dynamics, 11(1):165-188, 2017.

[16] R. C. Lyndon and P. E. Schupp. Combinatorial Group Theory. Springer Berlin Heidelberg, 2001.

[17] W. Magnus. Residually finite groups. Bulletin of the American Mathematical Society, 75(2):305-317, mar 1969.

[18] A. I. Mal'cev. On homomorphisms onto finite groups. Uchen. Zap. Ivanovskogo Gos. Ped.Inst., 1958.

[19] J. C. C. McKinsey. The decision problem for some classes of sentences without quantifiers. Journal of Symbolic Logic, 8(3):61-76, sep 1943.

[20] S. Meskin. A finitely generated residually finite group with an unsolvable word problem. Proceedings of the American Mathematical Society, 43(1):8-8, jan 1974.

[21] A. Mostowski. On the decidability of some problems in special classes of groups. Fundamenta Mathematicae, 59(2):123-135, 1966.

[22] E. Rauzy. Obstruction to a Higman embedding theorem for residually finite groups with solvable word problem. Preprint.

[23] A. M. Slobodskoi. Unsolvability of the universal theory of finite groups. Algebra and Logic, 20(2):139-156, mar 1981.

[24] J. Wilson and P. Zalesskii. Conjugacy separability of certain torsion groups. Archiv der Mathematik, 68(6):441-449, jun 1997.

Current address: Université de Paris. UFR de Mathématiques. Bâtiment Sophie Germain. 8 place Aurélie Nemours, 75013 Paris, France

Email address: emmanuel.rauzy.14@normalesup.org 